\title{
A construção da memória cultural em um contexto histórico mutante: a experiência do EILEALIVE
}

Paula Monseff Perissinotto ${ }^{1}$

\section{Introdução}

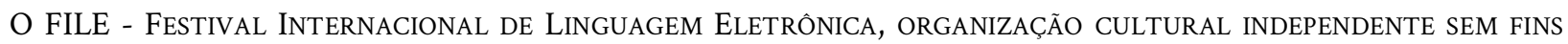
lucrativos, concebida em 1999, realizou sua primeira exposição no ano 2000, no Museu de Imagem e do Som (MIS), em São Paulo, Brasil. O nome "FILE”, que em português é traduzido como "arquivo", foi escolhido por fazer referência ao item no canto superior esquerdo no menu de quase todos os programas Windows ${ }^{2}$ e de outros sistemas operacionais. O festival exibia inicialmente apenas manifestações estéticas produzidas para a internet, mas com o passar do tempo seus conteúdos se bifurcaram em duas frentes principais: a on-line, com websites, bancos de dados, mídias sociais, editais anuais, etc; e a off-line, com exposições, publicações, simpósios, oficinas e performances. Até o momento, o festival organizou 49 exposições em 7 cidades diferentes.

Durante a chegada da internet banda larga no país, o festival contribuiu ativamente com o processo, expondo artistas vanguardistas que desenvolviam obras nesse suporte. Como resultado, a organização tornou-se uma plataforma cultural com visibilidade internacional, que segue mostrando obras de arte produzidas com recursos digitais como parte do entrelaçamento contínuo e sempre emergente entre a tecnologia e a arte. Todavia, a crescente sofisticação de algoritmos levou gradualmente ao surgimento de uma nova inteligência artificial nas redes e estar presente no mundo on-line agora requer o uso de algoritmos complexos e recursos financeiros significativos. O relacionamento on-line com o público, por sua vez, tornou-se dependente de sistemas sofisticados. Os programadores, que no passado se especializaram em apenas uma linguagem, trabalham atualmente em diferentes linguagens simultâneas.

A geração nascida no final do século XX, período em que computadores ainda eram muito raros no país, agora vive em uma sociedade em que tecnologias como a internet móvel são totalmente integradas na vida cotidiana. Uma das maiores conquistas do festival foi a sua audiência, que também se transformou ao longo do tempo. Milhões de pessoas no Brasil visitaram as exposições e os eventos realizados pelo festival. Neste momento, um dos desafios enfrentados é manter um diálogo com as novas gerações e, ao mesmo tempo, manter sua posição como uma organização que cria um ponto de conexão entre o artístico, o inovador e o histórico, olhando para o futuro e preservando o passado

Os registros desse percurso são documentos e dados, que contam a história dos eventos realizados por meio de textos bilíngues, palestras traduzidas, imagens e vídeos de performances e de obras de arte. Este conteúdo está em vários formatos,

\footnotetext{
${ }^{1}$ Cofundadora e organizadora e do FILE, Festival Internacional de Linguagem Eletrônica. Em 2020 ingressou como doutoranda na Universidade de São Paulo, Escola de Comunicações e Artes | ECA, em Poéticas Visuais. Membro do Grupo de Pesquisa Realidades licenciado pelo CNPq, liderado pela Profa. Dra. Silvia Laurentiz, que por sua vez, vincula-se formalmente a Escola de Comunicações e Artes e ao Departamento de Artes Visuais, ECA/USP. Contato: paula@file.org.br ${ }^{2}$ Dadas as características do universo tratado e a abordagem internacional do tema, o que reflete na frequência de termos estrangeiros nas discussões, e procurando preservar uma melhor organização do material tanto para especialistas quanto para o público geral, foram adotados os critérios da ABNT de destaque textual (itálico) para este artigo, salvo: nomes de empresas ou programas de conhecimento disseminado, como "Windows" ou "Excel", nomes de universidades e as siglas das instituições comentadas ao longo do artigo.
} 
totalizando publicações impressas, bancos de dados, DVDs de documentários, declarações de artistas, projetos técnicos e biografias, bem como registros formais e informais armazenados em estantes e caixas. Um dos principais compromissos do festival é fornecer ao público acesso gratuito a todas as informações sobre as obras de arte exibidas, os artistas que participam de cada evento e todo conteúdo gerado pela organização em ambiente acessível e on-line. A internet é construída sobre uma dinâmica complexa e viva, e esse processo exige atualização constante.

Dessa maneira, um dos objetivos do FILE é disponibilizar publicamente os documentos e materiais que foram arquivados em um único ambiente confiável on-line e off-line, para ser compartilhado com estudiosos, pesquisadores, artistas, curadores e interessados no campo da arte e tecnologia. Nesse sentido, a organização do festival busca parceiros institucionais para seguir a missão de preservar os traços deixados pelos 20 anos de trabalho e institucionalizar um legado, além de, ao mesmo tempo, aprofundar e fortalecer o relacionamento entre arte e tecnologia no âmbito internacional, tendo o festival sido criado por uma iniciativa brasileira.

O ARQUIVOVIVO - encontros on-line realizados pelo FILE nos dias 29, 30 e 31 de março de 2021, que contaram com profissionais e pesquisadores dedicados às áreas de memória digital, preservação do patrimônio cultural e tecnologia da informação, presentes em seis sessões que apresentaram estudos de caso, exemplos de arquivos e estratégias de conservação para acervos que visam à difusão livre e à proteção de coleções de arte e tecnologia - teve desdobramentos práticos, sendo que aqui trataremos especificamente dos desdobramentos da sessão intitulada “Arquivo como Instituição”, realizada em 30 de março de 2021, e da sessão “O que arquivar? Demandas e princípios da memória digital”, realizada no dia 31 de março de2021, ambas disponíveis on-line $e^{3}$.

A partir da sessão “Arquivo como Instituição”, no sentido de se estabelecer um plano para conectar os respectivos arquivos, uma aliança de parceiros foi efetivada entre a equipe do ISEA (Simpósio Internacional de Arte Eletrônica), do ACM SIGGRAPH (Grupo de Interesse Especial da ACM em Gráficos e Técnicas Interativas) e do FILE (Festival Internacional de Linguagem Eletrônica). A sessão apresentou os representantes do ISEA e do ACM SIGGRAPH, onde Wim van der Plas discorreu sobre os arquivos do ISEA, Janice Searleman apresentou os arquivos SIGGRAPH e Bonnie Mitchell apresentou um plano para conectar os arquivos ISEA e SIGGRAPH. Paula Perissinotto, como representante do FILE, apresentou os planos atuais para o desenvolvimento do acervo digital do festival on-line.

Em 1988, foi realizado o primeiro ISEA (International Symposium on Electronic Art), no qual diversos pesquisadores e artistas da área perceberam-se voltados para problemáticas semelhantes e decidiram renovar o encontro dois anos depois. O ISEA, segundo encontro, contou com alguns participantes que queriam realizar o evento na Austrália. Assim, o terceiro encontro foi realizado em Sydney, o quarto em Mineápolis e, no ano seguinte, em Helsinki. Atualmente, a organização já realizou 26 simpósios ao redor do mundo, tendo visitado países como Colômbia, África do Sul e Coreia. No ano passado, a edição foi realizada virtualmente a partir de Montreal, Canadá.

Nesse sentido, Wim van der Plas, fundador do ISEA, considera que a organização surgiu de forma anárquica, espontânea e desorganizada. O sociólogo inicialmente apenas tentava coordenar a realização do simpósio. Em 2006, uma fundação foi criada, baseada em uma organização de membros filiados de 1990, para cuidar da coordenação do evento. No entanto, essa fundação não recebe financiamento e por isso não é uma organização central no processo. Percebendo a demanda por registros do que era discutido e exibido nos eventos, o arquivo do ISEA foi concebido com o intuito de reconstruir a história perdida dos primeiros anos da instituição.

O ISEA é predominantemente uma conferência acadêmica que apresenta eventos artísticos paralelos, que acabam por tornar-se mais importantes do que o viés acadêmico. Seu arquivo on-line é baseado em WordPress e possui um menu central com uma seção "About", que trata da missão do evento, apresenta notícias e um glossário com os termos que são utilizados, bem como a listagem de equipes voluntárias nacionais e internacionais que contribuem com o arquivo. As atividades,

${ }^{3}$ FILEALIVE / ARQuivovivo, 2021a, 2021b. 
catálogos e programações são diferenciados entre si. Ao abrir uma edição específica do simpósio, uma página com informações básicas é apresentada, contendo equipes, programação, comitês que realizam revisões por pares, júris que avaliam e selecionam as obras, temas e parceiros da edição. O conteúdo da edição é dividido em três frentes: a acadêmica, que pode ser encontrada em "Presentations"; a educacional, encontrada em "Workshop"; e a artística, encontrada em "Art Events". As páginas individuais de obras contêm um pequeno resumo, vídeos, imagens e biografia do artista.

O arquivo apresenta também informações sobre o ISEA Summit, ocorrido em 2020, que teve como objetivo estimular a cooperação entre arquivos de arte digital e mídia arte, do qual a iniciativa dos encontros on-line FILEALIVE / ARQUIVOVIVO é decorrente. Outra seção interessante do arquivo é "Other New Media Art Archives", onde podem ser encontradas instituições e arquivos da área. A aba principal é "Symposia", onde os eventos podem ser encontrados por ordem cronológica. Por fim, há a seção "Proceedings/Catalogues", que apresenta publicações e catálogos criados ao longo dos anos. Muitas páginas não possuem imagens ou vídeos e não refletem o espírito inspirador do simpósio, assim como não representam os acontecimentos incríveis realizados ao longo dos anos. Nesse sentido, a ideia de construir um novo arquivo ISEA, mais visual, dinâmico e funcional, surgiu de Bonnie Mitchell. As categorias base utilizadas no ISEA Symposium Archives foram as mesmas, no entanto, organizadas em uma nova hierarquia. Também foram adicionadas as categorias "Contributors" e "Visualization", que possibilita a visualização de dados sobre participantes, edições e obras. A página "Persons" também foi uma atualização realizada no site e disponibiliza informações sobre os participantes ao longo do tempo, não limitada a apenas uma edição. Esse arquivo apresenta recursos mais complexos de interconectividade, nos quais dados e navegação podem ser organizados de diversas formas.

Por outro lado, a nova versão do arquivo também apresenta problemas. O início anárquico da instituição se deu em um momento propício e serviu a um movimento que se tornou continuado, percorrendo cidades por todo o mundo, o que significou reinventar a roda em todas as edições, apesar do conhecimento acumulado. Para organizar o imenso conteúdo produzido, as equipes que tiveram participação no processo usaram termos e categorias diferentes, o que atrapalha a elaboração de um arquivo bem estruturado. Acrescentar novos artistas ou contribuidores à nova versão do arquivo exige o preenchimento de formulários complexos e atualizações individualizadas. Bonnie Mitchell e Janice Searleman, que exercem atividade voluntária, estão trabalhando atualmente para tornar esses processos menos árduos.

Janice Searleman é professora adjunta de pesquisa na Clarkson University, membro do Comitê de História e Arte Digital do SIGGRAPH, além de codirigir o Arquivo Digital ISEA, com Bonnie Mitchell e Wim van der Plas. Sua apresentação tratou do Art Show Archive ACM SIGGRAPH, também codirigido por ela. Quando se aposentou em 2015, após ensinar Ciência da Computação por 37 anos, a arquivista passou a integrar o comitê de arte digital do ACM SIGGRAPH, onde conheceu Bonnie Mitchell, que já desenvolvia projetos na área de arquivamento anteriormente. As pesquisadoras uniram forças em prol da criação de arquivos que fossem compostos por interconexões de dados mais complexas. Em 2019, também passou a trabalhar na versão atual do Arquivo Digital ISEA. Ambos os arquivos possuem configurações semelhantes e os mesmos back-end. A ACM - Association for Computing Machinery's - é uma associação profissional e acadêmica para pessoas de todas as áreas da computação. Já o SIGGRAPH - Special Interest Groupon Computer Graphics and Interactive Techniques - é para profissionais que trabalham e se concentram nas áreas de computação gráfica e interatividade. A organização tem promovido conferências anuais ao longo de quase 50 anos e, em 1981, incorporou um show de artedigital à sua programação. O arquivo digital on-line disponibiliza as edições das conferências, publicações, apresentações e eventos relacionados, de 1981 até o presente. O arquivo também inclui o SIGGRAPH Asia e as exposições da Comunidade de Arte Digital (DAC) on-line. O menu principal contém as seguintes seções: Art exhibitions, Contributors, Artwork, Writings \& Talks, Collections, Visualization e About us. É possível navegar cronologicamente, alfabeticamente, por categorias técnicas ou conceituais, por imagens e por texto. A visualização pode ser feita porlistagens ou galerias e as publicações podem ser encontradas em PDF e também são linkadas à tabela digital do ACM SIGGRAPH. 
A seção de coleções é uma das mais inovadoras, disponibilizando curadorias de colaboradores.O exemplo citado durante a apresentação é de Douglas Dodds, responsável por criar uma coleção contendo obras do SIGGRAPH que estão em exposição atualmente também no Victoria \& Albert Museum, onde écurador sênior. As páginas individuais de coleção possuem informações básicas como título, tema, imagem, descrição e também links que redirecionam o visitante a instituições pertinentes ou correlacionadas àquela coleção. Interessados em disponibilizar parte do arquivo em Open Source, o segmento "Visualization" foi adicionado, possibilitando que dados sobre obras e apresentações sejam vistos e baixados em formato CSV. O filtro de pesquisa da seção possui categorias como gênero, cidade, estado, coordenadas, e-mail, site, apresentações, cargo do trabalho, etc. O objetivo é disponibilizar esse material para artistas que podem potencialmente reinterpretar os dados e criar novas propostas artísticas. $\mathrm{O}$ arquivo também conta com um mapa de localização que indica as coordenadas de contribuidores e participantes, como artistas e palestrantes que forneceram essas informações ao longo do tempo.

Bonnie Mitchell é artista e codiretora dos Arquivos ISEA e SIGGRAPH, além de professora na Bowling Green State University em Ohio, EUA, e tem se concentrado em estudos de interconectividade para arquivos. Em sua participação, iniciou esse trabalho realizando testes de back-end no Arquivo do SIGGRAPH e exportando o modelo para o Arquivo do ISEA. Seu intuito é expandir a ideia da interconectividade entre arquivos globalmente.

Para interconectar bancos de dados digitais é necessário observar quais são as diferenças significativas que apresentam, bem como levantar a questão: “O que será conectado?”. Ao escolher categorias predeterminadas, como, por exemplo, "pessoas", partes de informações contidas no arquivo relacionadas a essa categoria serão conectadas, como eventos em que tiveram participação, obras que realizaram, etc. Nesse sentido, é necessário visualizar como essas conexões serão desenhadas. A pesquisadora nomeou esses pontos centrais para a interconexão de "pods", sendo possível por meio deles criar um sistema de gerenciamento de conteúdo personalizado, observando taxonomias de relacionamentos entre categorias e listas expansíveis.

Nesse sentido, as páginas dos Arquivos ISEA e SIGGRAPH são criadas como campos bidimensionais a partir das conexões feitas entre "pods". Essa ideia de categorização é fundamental para criar interconexões. Umas das dificuldades envolvidas nesse processo é que artistas de arte digital costumam criar novas categorias, ou ainda desenvolvem obras que entram em diversas categorias ou nenhuma delas. Com o surgimento da arte em telefones celulares, por exemplo, essa categoria foi criada e tagueada como “mobiles". Dessa forma, uma página passou a mostrar todos os resultados de informação daquela nova tag e o código ficou responsável por unir todo o conteúdo relacionado em um só lugar.

Ao comparar os acervos dos Arquivos ISEA e SIGGRAPH, foi possível mapear informações que eram disponibilizadas por apenas um dos arquivos. Para que absorvessem conteúdos um do outro, surgiu a ideia de criar uma conexão. No fim das páginas, foram incluídos ícones que guiam os visitantes a arquivos que têm informações adicionais sobre um artista em comum ou trabalhos em comum. Coleções e museus também foram linkados. Em uma experiência recente, Bonnie Mitchell e Janice Searleman começaram um novo repositório para arquivar a história do SIGRAAPH e suas conferências, e para isso o arquivo anterior teve que ser absorvido, criando uma fusão de conteúdos. Foram definidas duas possibilidades para realizar esse processo: um sistema descentralizado, no qual os arquivos absorvem dados um do outro; ou um sistema centralizado, em que um $H U B$ central é acessado por ambos os arquivos e no qual dados são exportados e absorvidos.

Ao expandir esse conceito para uma rede global de arquivos (Figura 1), Bonnie destacou problemas de propriedade intelectual em potencial, dado que apenas um evento teria permissão para usar informações e materiais disponibilizados pelos artistas, e levantou as questões: "Quem poderia gerir essas licenças? Um HUB central poderia fragilizar a rede de arquivos e favorecer ataques em massa, além de que esses arquivos de grande e pequeno porte são planejados com diferentes back-ends”. Para iniciar essa conciliação, Wim van der Plas começou um levantamento de arquivos, que deve ser continuado, e um consórcio deve ser estabelecido entre algumas instituições, tais como: ISEA, o ZKM, o ADA, o FILE, entre outros, para que haja comunicação efetiva entre os arquivos. Este projeto já se encontra em andamento desde o ISEA 2019, realizado no Asia Culture Center. 


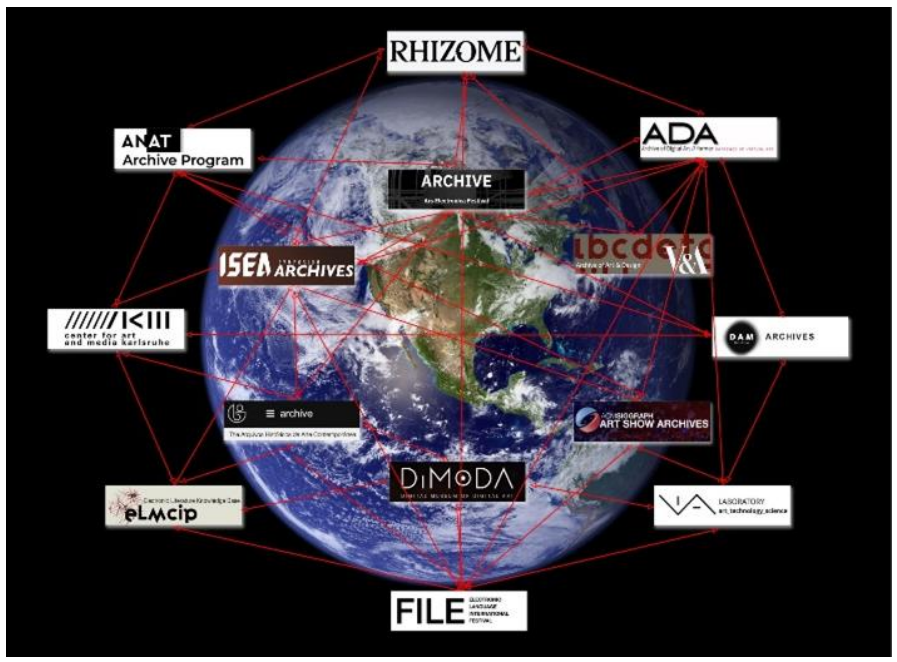

Fig. 1 Referência sobre a possibilidade de conexão entre os arquivos da área ${ }^{4}$.

\section{O Arquivo do EILE EESTIVAL}

O arquivo do FILE teve início em 2012 com Gabriella Previdello, numa organização física de fichas de inscrições e informações enviadas ao festival pelos artistas, através do correio, desde o ano 2000. Recentemente esta organização foi retomada por Paula Perissinotto como objeto de pesquisa de doutorado na área de Poéticas Visuais da ECA - Escola de Comunicação e Artes da USP, sob orientação da pela professora doutora Silvia Laurentiz, para encontrar uma forma de organizar o conteúdo de 20 anos do festival, tanto de forma física como digital, de modo a conectá-lo com instituições no mundo que atuam no campo da arte e tecnologia por meio de uma rede cooperativa. A qual, atualmente, conta com um time envolvendo três instituições (Tainacan, Percebe e FILE) e o Grupo de Pesquisa Realidades, licenciado pelo CNPq e liderado pela Silvia Laurentiz, que, por sua vez, vincula-se formalmente à Escola de Comunicações e Artes (ECA/USP) e ao Departamento de Artes Visuais da mesma escola.

Antes da proposta de integrar-se a outras instituições, o FILE festival já pretendia reorganizar o seu repositório digital e mudar a estrutura incialmente instalada no WordPress para torná-la interconectada entre si. O caminho planejado pretendia utilizar um gerenciador de conteúdo com a tecnologia headless, como, por exemplo o Strapi5.

A tecnologia headless, sugerida pela equipe de programadores do festival, prevê que uma das camadas que normalmente estaria junta à aplicação, não exista, ou seja, a aplicação só possui o back-end, ficando a cargo de quem usar o software montar seu front-end conforme sua necessidade. E é exatamente esse o papel que hoje softwares com APIs fazem: fornecem endpoints seguindo a ideia da arquitetura de microservices, forçando as interfaces visuais separadas a consumir esses web services e se beneficiar deles de forma independente e desvinculada. Os benefícios são diversos, pois essa tecnologia possibilita uma liberdade de personalização imensa e o uso em diversos canais de comunicação, ou seja, uma comunicação multicanal.

Desta forma, a estrutura do repositório digital seria definida dentro do gerenciador de conteúdo de forma semelhante a uma planilha Excel, apresentando colunas e tipos de dados. Uma vez estruturados, os dados inseridos no gerenciador de conteúdo se tornam disponíveis através de APIs e ficam acessíveis publicamente em endereços de links diretos da aplicação.

\footnotetext{
${ }^{4}$ Filealive / Arquivovivo, 2021a, 2021b. Apresentação no Arquivo Vivo - encontros on-line de Bonnie Mitchell, na sessão “Arquivo como instituição", em: 30, mar. 2021.

${ }^{5}$ Disponível em: <https://strapi.io/>. Acesso em: 30, set. 2021.
} 
Esse formato é tendência na tecnologia atual, sendo largamente utilizado em aplicativos web modernos criados em javascript, como o React, o Gatsby e o Next.

Porém, uma outra possibilidade surgiu a partir dos encontros on-line decorrente da sessão "O que arquivar? Demandas e princípios da memória digital”, e trata-se do projeto Tainacan, abordado por seu coordenador, Dalton Martins, em sua apresentação. Conceitualmente, o software Tainacan é muito semelhante ao gerenciamento de conteúdo headless, mas utiliza o banco de dados WordPress, no qual o acervo do FILE já se encontra hospedado.

O Tainacan é produto de um trabalho desenvolvido na faculdade de Ciência da Informação da Universidade de Brasília, onde se alocam os cursos de arquivologia, museologia, biblioteconomia e um programa de pós-graduação, configurando um espaço propício para o desenvolvimento e exploração de questões em torno da memória, dos acervos e dos arquivos. Dalton Martins é também coordenador do Laboratório de Inteligência de Redes, criado entre 2018 e 2019, que tem como objetivo fomentar a prática e a experimentação na perspectiva dos acervos digitais, da ciência de dados, da aprendizagem de máquina e do desenvolvimento de análises de técnicas aplicadas a arquivos, buscando a criação de tecnologias e conhecimento nesse âmbito.

O nome do projeto, que tem origem indígena, foi escolhido tanto pelo seu potencial de representatividade quanto pela simbologia que carrega, dado que se refere à deusa dasconstelações para os povos tupi-guarani. Aplicada aos arquivos, a palavra reflete a criação de constelações de dados e a sua complexa união. O nome surgiu da ligação do projeto com a Universidade Federal de Goiás, que possui um núcleo de formação de professores indígenas e que forma, junto com a UNB e o Instituto Brasileiro de Informação em Ciência e Tecnologia (IBICT), a tríade de sedes do Tainacan. O projeto teve início em 2014, como elemento técnicoe acadêmico integrante de uma política nacional de acervos digitais do Ministério da Cultura. Dalton Martins traçou em 2020, junto a uma pesquisadora de mestrado, a história de tentativas de criação de políticas nacionais sobre o digital, a partir de diagnósticos que mostraram que essa ainda é uma dimensão dispendiosa, muito distante e difícil de introduzir nas instituições brasileiras. Nesse sentido, havia a demanda por uma política nacional cultural que gerasse soluções de baixo custo e por práticas essenciais para a interconexão de dados de acervos e arquivos, como, por exemplo, hospedagem de dados em nuvens, softwares livres de boa qualidade, cursos gratuitos para instituições, modelos de dados, vocabulários controlados, ontologias, estratégias de catalogação, etc. Para atingir tal objetivo, é fundamental que essas implementações sejam coletivas, com a participação da sociedade civil e entes governamentais. Desde a concepção da ferramenta, o cenário político e cultural sofreu diversas mudanças que afetaram esse processo. Dessa forma, o projeto teve que mudar de foco e começar a formar parcerias com instituições.

O Tainacan, baseado em WordPress, está em uso e é utilizado de diversas formas por instituições centrais na preservação da memória nacional. No caso do Instituto do Patrimônio Histórico eArtístico Nacional (IPHAN), por exemplo, a ferramenta foi utilizada para o desenvolvimentodo inventário do patrimônio imaterial. No Instituto Brasileiro de Museus (IBRAM), é utilizadano gerenciamento de acervos digitais ligados aos museus, e na Fundação Nacional de Artes FUNARTE), organiza acervos de teatros brasileiros e museus das artes.

O objetivo do software, desde a sua concepção, foi tornar-se uma ferramenta de fácil acesso, implementação e interação, dado que foi pensado para a realidade e problemáticas brasileiras. Originalmente, quando a discussão sobre modelos de metadados foi iniciada no país, não houve padronização. É um desafio abarcar a diversidade de objetos e formatações escolhidas pelas instituições. O software pretende, nesse sentido, facilitar a criação de padrões de forma flexível, não adotando um modelo de dados específico.

Em prol dessa meta, o programa deveria ser uma tecnologia não apenas técnica, mas também socializável, capaz de produzir conhecimento em rede e detentora de uma base de usuários bem estabelecida. Para isso, a equipe desenvolve ações de curadoria essenciais ao trabalhar com objetos digitais, como: a criação de agrupamentos e coleções; a criação de metadados a partir da descrição e documentação de objetos; a adoção de vocabulários controlados e taxonomias que possam classificar e indexar itens; a disponibilização de diferentes filtros para gerar formas multifacetadas de navegação e de interconexão de dados; a criação de redes de relacionamentos semânticos e conexão entre coleções e objetos; a edição dos itens digitais em si, como 
arquivos, links e conteúdo; e a produção de exposições contextualizadas, que sejam acessíveis e funcionem bem em diferentes dispositivos e padrões de tela.

Para isso, a equipe decidiu fazer uso de repositório digital em software livre preexistente, o WordPress. Para entender a aplicabilidade de sistemas de gerenciamento de conteúdo na internet, Dalton Martins recomenda o acesso ao W3Techs, site que fornece estatísticas atualizadas sobreesses sistemas usados internacionalmente. O WordPress é base de 40,5\% do gerenciamento de conteúdo on-line atualmente. Logo, é uma tecnologia socializada, conhecida e disseminada, e, por esses fatores, a escolhida para o desenvolvimento do plugin e da interface Tainacan.

O software já foi baixado mais de 8 mil vezes desde que sua distribuição foi iniciada pelo repositório de plugins do WordPress, possui mais de 300 instalações ativas de instituições, sendo mais de 20 universidades públicas, como a Universidade Federal do Rio Grande do Sul, Universidade Federal de São Carlos, Universidade Federal de Minas Gerais, Universidade de Brasília, Universidade de São Paulo e Universidade Estadual de Campinas, entre outras. Pela sua versatilidade, o software também é utilizado por instituições culturais privadas. Nesse sentido, o FILE festival reconsiderou a migração dos dados digitais para o sistema headless STRAPI e optou pela organização do banco de dados on-line utilizando a tecnologia do Tainacan, compatível com o ambiente WordPress em que os dados do festival já se encontram hospedados.

Inicialmente foi feito juntamente com a equipe da empresa Percebe um diagnóstico do esquema de organização e catalogação dos dados do conteúdo digital do FILE para então se desenhar a arquitetura informacional através de levantamento das tipologias informacionais, características dos acervos e necessidades de modelagem informacional institucionais, já considerando as estratégias de interconexão com os arquivos ISEA/ SIGRAAPH.

Para a realização dessa etapa de diagnóstico, a equipe do FILE forneceu à equipe da Percebe a estrutura interna (Figura 2) utilizada atualmente para catalogar os conteúdos do FILE Festival a serem migrados, além das referências atuais da estrutura utilizada pelo Arquivo Digital ISEA, fornecida pela sua codiretora Bonnie Mitchell. A ideia é se adaptar a essa estrutura de catalogação para possível interoperabilidade entre sistemas. 


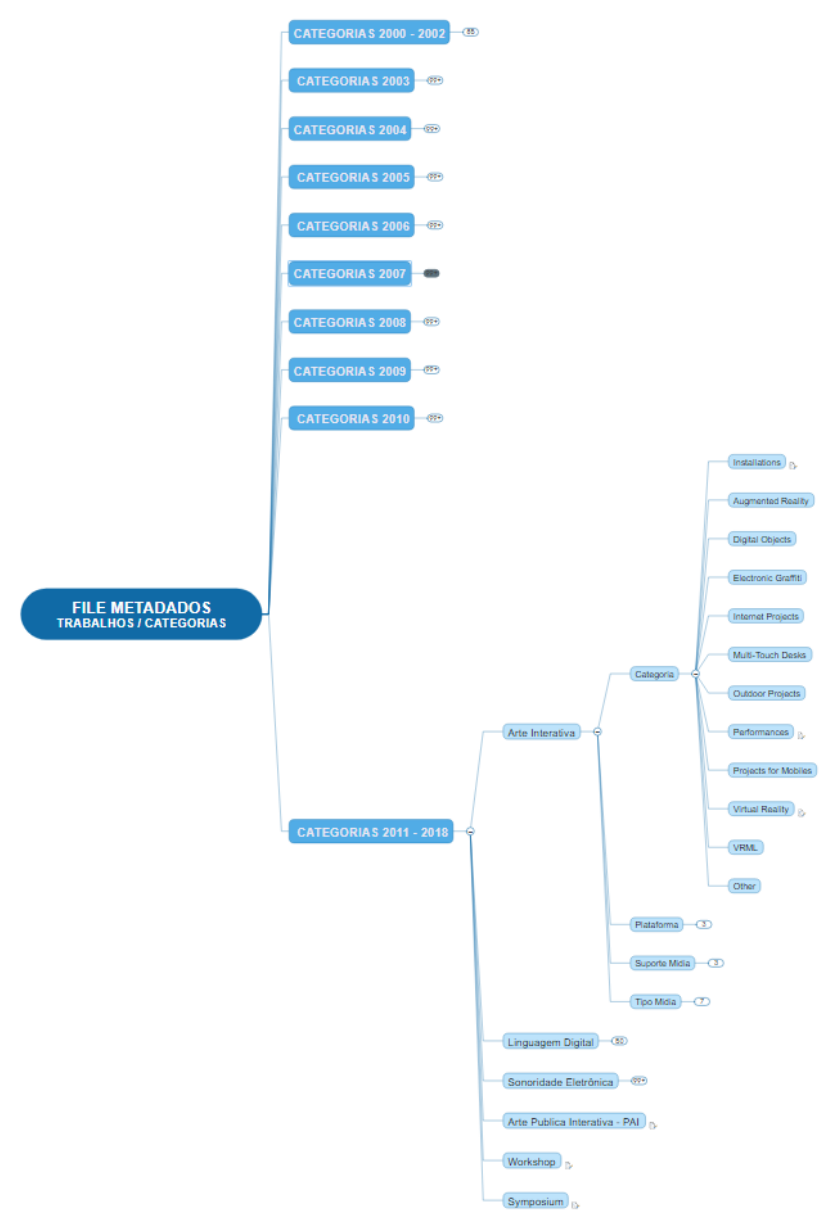

Fig. 2 Referência ao diagnóstico realizado pela Percebe das categorias do FILE para uma planilha onde foram organizadas por níveis de termos. Após a sistematização, foram analisados de forma descritiva ${ }^{6}$.

Para facilitar a análise e diagnóstico, tanto os dados relativos às referências de categorias, como de metadados e o mapa de organização de informações/dados utilizados pelo FILE foram transpostos de um modelo hierárquico em árvore, conforme Figura 2, para uma planilha, onde foram organizados por níveis de termos. Após esta sistematização, os dados foram analisados de forma descritiva (Figuras 3 e 4). Foram então identificados nas referências internas três conjuntos informacionais que devem ser trabalhados para o repositório digital no Tainacan:

- Categorias: tema, formato, tópico, gênero, plataforma e mídia;

- Tipologia de documentos: catálogos, logomarcas, projetos realizados, projetos não-realizados, administrativo, modelos;

- Metadados: campos descritivos atualmente em uso no site do FILE.

\footnotetext{
${ }^{6}$ Modelo hierárquico das categorias do FILE em árvore, cedido à autora por Fabiana Krepel, 2020.
} 


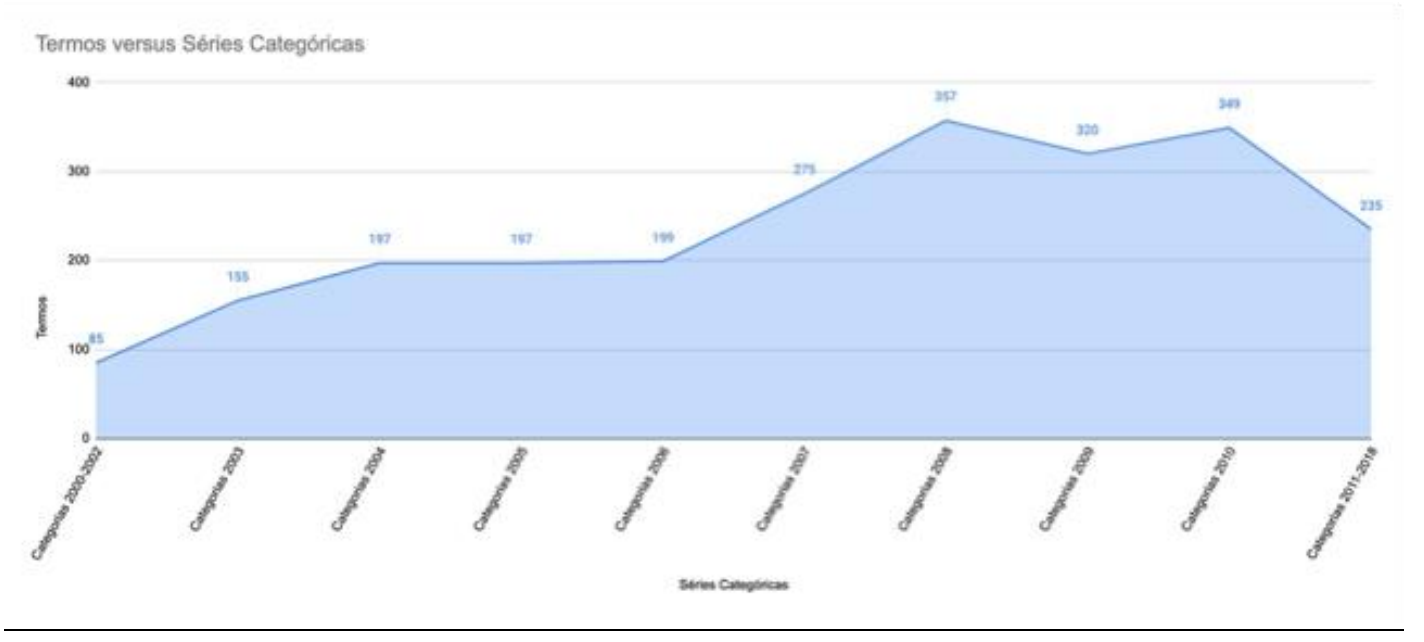

Fig. 3 Gráfico criado pela Percebe em 2021 a partir das referências das categorias para comparar a relação dos termos versus categorias criadas pelo FILE $^{7}$.

Evoluçäo Termos X Niveis (2000-2018)

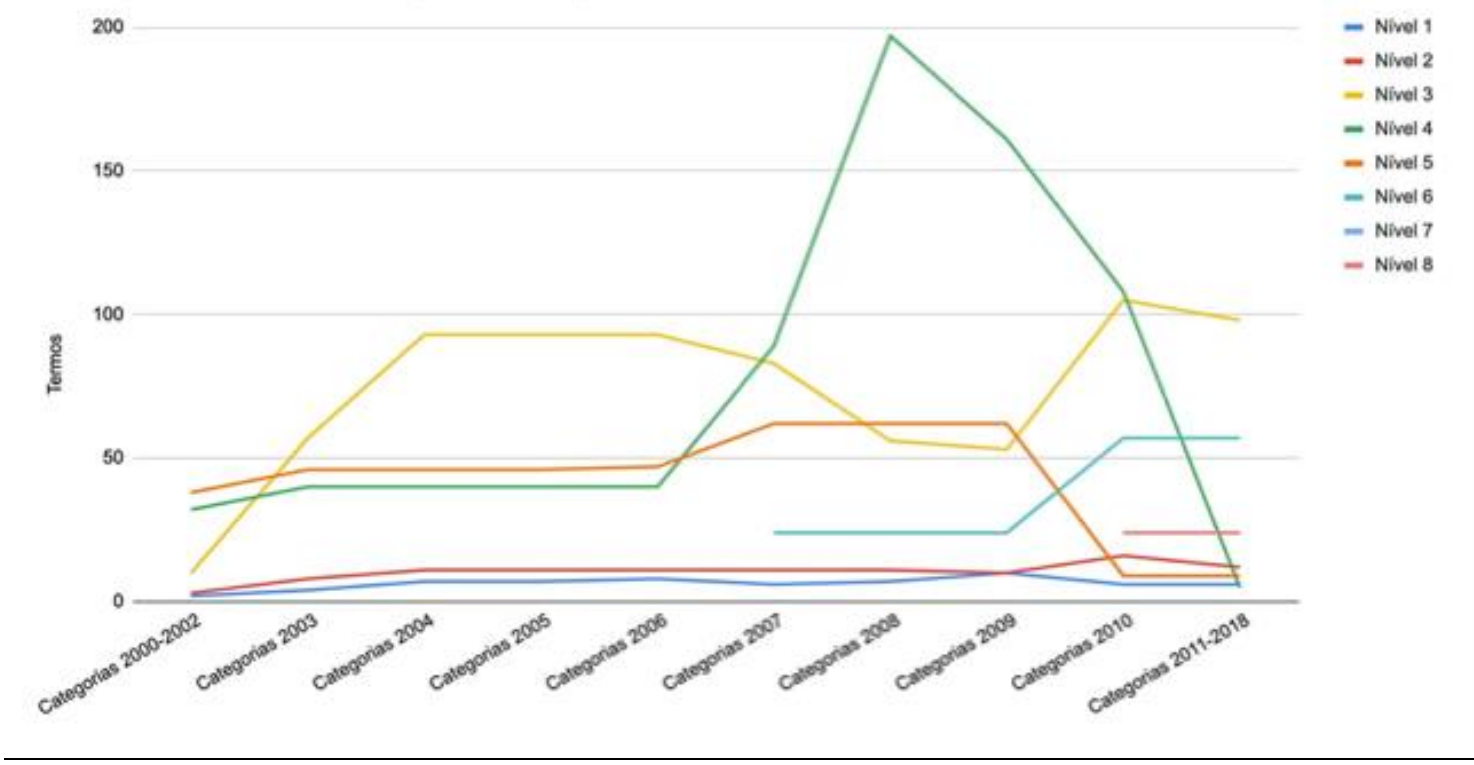

Fig. 4 Gráfico criado pela Percebe em 2021 para ilustrar a evolução dos termos versus níveis referentes aos anos de 2000 a 2008 do arquivo do FILE ${ }^{8}$.

${ }^{7}$ Gráfico cedido à autora pelo FILE.

${ }^{8}$ Idem. 


\section{Termos versus Ocorrências}

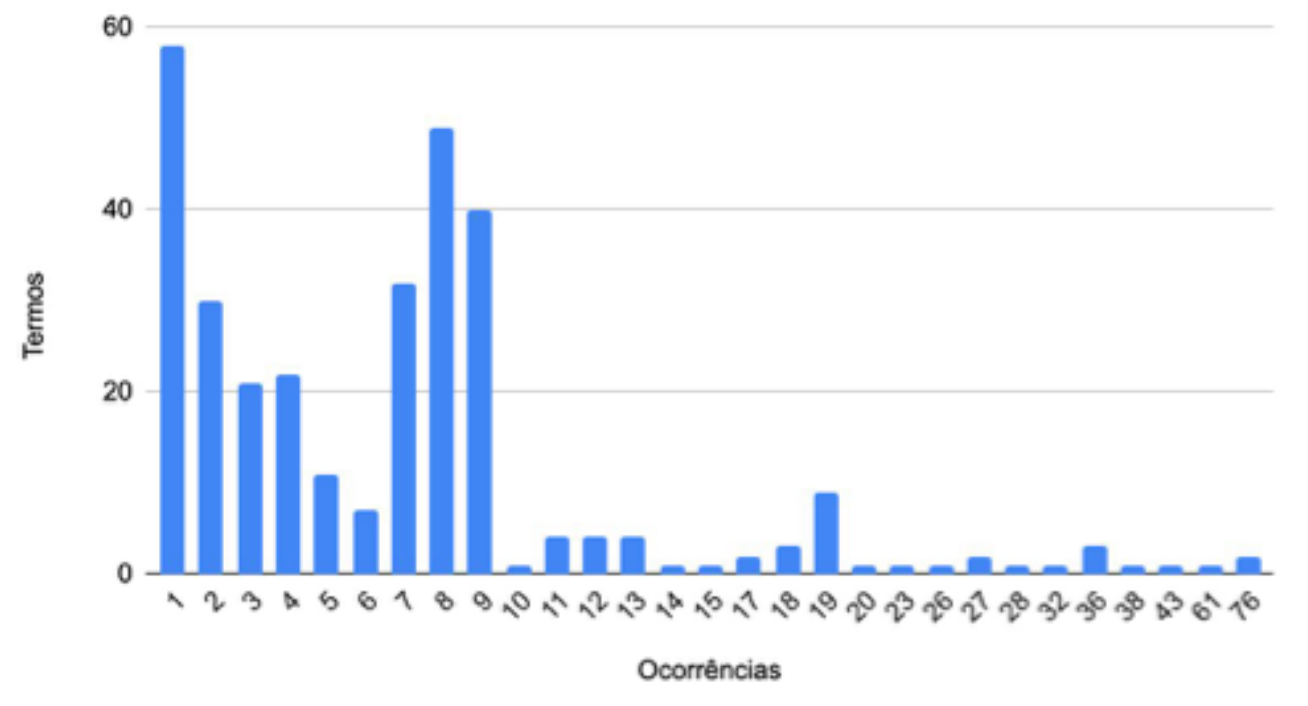

Fig. 5. Gráfico criado pela Percebe em 2021 para identificar a ocorrências dos termos usados pelo FILE ao longo do período de 2000 a $2018^{9}$.

Foram identificados 314 termos utilizados no total em toda série categórica histórica do FILE, sendo que há termos que apareceram ao menos 10 vezes, que são os termos localizados mais ao fim das hierarquias e que, portanto, aparecem em várias categorias simultâneas.

A partir da análise de categorias, sugeriu-se uma série de procedimentos que podem ser aplicados a elas atualmente:

$1^{\circ}$ Normalização: há nomes parecidos, com diferenças de formas de escrita, pontuação ou espaçamento que podem ser padronizados;

$2^{\circ}$ Definir uma tipologia de vocabulários controlados e concentrar a dispersão dos termos em torno deles, como Tema, Formato, Mídia, Plataforma, Gênero, Tópico;

3o Mapear os vocabulários para termos utilizados em bases semânticas como Wikidata e AAT do Getty, quando existentes;

$4^{\circ}$ Os vocabulários podem ser usados em diferentes tipos de metadados.

$\mathrm{Na}$ análise do mapa de organização, foi identificado que para cada tipo de documento, há até 9 subníveis de detalhamento de sua tipologia, e que há a mistura entre tipos de documentos, datas e eventos. Também foi sugerido que a tipologia de documentos identificados no mapa de organização aponta para a necessidade de apenas uma única coleção de documentos e diferentes vocabulários controlados para sua classificação.

Comparativamente às referências fornecidas pelo ISEA, pôde-se observar que os metadados que utilizam são bem mais detalhados e definidos do que os atualmente em uso pelo FILE (FILE: 19 metadados (5,3\% do ISEA); ISEA: 355 metadados), e as taxonomias são em menor quantidade e com um número muito menor de termos (FILE: 314 termos; ISEA: 54 termos $(17,2 \%$ do FILE))

${ }^{9}$ Idem. 
A partir dos resultados obtidos neste diagnóstico, o FILE está desenvolvendo e realizando a disponibilização de seu arquivo dos anos de 2015 a 2019, através do repositório digital Tainacan, instalado no WordPress, e de acordo com o desenho da arquitetura informacional e a modelagem semântica propostos para a organização de suas coleções.

A instalação final do Tainacan incluirá a configuração, revisão e parametrização do modelo de dados e instalação do design no programa. Será realizada a adaptação e implementação do tema do Tainacan, visando a configuração do software em consonância com o modelo de comunicação visual adotado pelo FILE

Após as etapas de configuração, implementação, modelagem e desenvolvimento de interfaces do repositório, contaremos com a capacitação da equipe do FILE, através de uma oficina para utilização do Tainacan e para dar início ao input de dados e informações relativos aos anos de 2015 a 2019. O desenvolvimento do projeto está sendo realizado através de uma série de encontros on-line ao longo do ano de 2021.

\section{Os Arquivos do ACM SIGGRAPH / ISEA}

O desenvolvimento inicial do Arquivo ISEA Clássico (http://isea-archives.org) começou em 2012 com uma conversão do arquivo original e um foco na construção de uma arquitetura de informação e na inserção dos dados. Wim van der Plas, cofundador do ISEA, vem acumulando e digitalizando artefatos e adicionando-os ao Arquivo Clássico. Depois de construir e preencher esse arquivo, percebeu-se que era necessário um sistema mais robusto para lidar com milhares de imagens e a interconexão dos dados. Portanto, embarcaram na construção de um sistema inovador que resultou nos novos Arquivos do Simpósio ISEA, que está alojado no servidor SIGGRAPH ${ }^{10}$.

O ACM SIGGRAPH Art Show Archive foi desenvolvido inicialmente como sites individuais para cada ano, dirigido por Bonnie Mitchell com a ajuda de seus alunos. Isso era insustentável devido à quantidade crescente de dados e também por não fornecer acesso fácil às informações ao longo dos anos. Jan Searleman e Bonnie Mitchell iniciaram um novo arquivo em 2014 com a ajuda de alunos e, em 2021, eles ampliaram seu escopo para incluir animações, tecnologias emergentes, cursos e outros materiais do SIGGRAPH ${ }^{11}$.

Ambos, os novos arquivos do ISEA e SIGGRAPH, foram construídos usando PHP, PODs, HTML e codificação CSS customizados e construídos sobre um front-end WordPress. A programação dos recursos avançados foi supervisionada por Jan Searleman e Bonnie Mitchell com alunos da Bowling Green State University (BGSU). O processo de preencher o arquivo pegando dados do Arquivo clássico ISEA foi massivo e estudantes e voluntários de todo o mundo lideraram o esforço. A coleta de dados para o Arquivo SIGGRAPH envolveu a digitalização de milhares de documentos e o trabalho com pioneiros na área. Ambos os novos arquivos permitem o acesso fácil e gratuito a uma incrível riqueza de material, permitindo que a próxima geração se beneficie e se inspire na criatividade e na pesquisa inovadora do passado.

Os arquivos ISEA e SIGGRAPH contêm milhares de inscrições mostrando o desenvolvimento e a importância da nova arte de mídia nos últimos 40 anos. Incluem informações sobre eventos de arte, apresentações de trabalhos acadêmicos, painéis, mesas redondas, palestras de artistas, workshops, animações e muito mais.

\footnotetext{
${ }^{10}$ Disponível em: <https://isea-archives.siggraph.org $>$. Acesso em: 20, set. 2021.

${ }^{11}$ Disponível em: <https://history.siggraph.org $>$. Acesso em: 20, set. 2021.
} 


\section{considerações finais - Interconexão prática entre os arquivos do ISEA, ACM SIGRAAPH e EILE festival}

Depois da implementação do arquivo do FILE Festival de acordo com uma modelagem semântica e de dados definidos, com os dados cadastrados relativos às informações dos anos de 2015 a 2019 poderemos considerar a realização da interconexão entre os arquivos do FILE e os do ISEA e SIGGRAPH. A proposta não é em hipótese alguma absorver os dados de um arquivo para outro, mas apenas exibir e vincular os dados localizados em cada um. Isso permitirá que os usuários percorram cada arquivo livremente para descobrir novas informações, aumentando assim a utilidade de cada arquivo, além de inserir uma iniciativa brasileira na construção da história da arte e tecnologia.

Os arquivos ISEA e ACM SIGGRAPH são construídos usando um back-end em WordPress com uma estrutura de gerenciamento de conteúdo personalizada chamada PODS. Este sistema permite que todos os campos de dados possam ser conectados a outros campos de dados e modelos, por meio do uso de taxonomias e relacionamentos bidirecionais. Este sistema também é desenvolvido através do uso de programação personalizada de modelos, funções de tema e código personalizado dos PODS. Tal sistema permitiu a exportação de dados e atualmente trabalha na sua importação.

No sentido de conectar novos arquivos de mídia em todo o mundo, essas são as etapas preliminares necessárias. Desta forma, ISEA e SIGGRAPH estão trabalhando na conexão de informações relacionadas a um colaborador individual do SIGGRAPH a informações sobre esse mesmo colaborador nos arquivos do ISEA, e vice-versa. As informações sobre uma mesma obra de arte serão conectadas, então, entre os arquivos. E, além disso, como tanto o ISEA quanto o SIGGRAPH residem no mesmo servidor, os problemas de segurança serão minimizados. Depois que a conexão de informações relacionadas a pessoas e eventos de arte no SIGGRAPH e no ISEA estiver funcional, os arquivos estarão prontos para começar a criar conexões bidirecionais com o arquivo do FILE.

Como apontado, o arquivo do FILE Festival está sendo desenvolvido usando a plataforma WordPress com a instalação do repositório digital Tainacan. O Tainacan é uma solução tecnológica para a criação de coleções digitais na Internet e contribui para a preservação e comunicação da produção cultural na internet, por meio da gestão e compartilhamento de acervos.

Uma vez que for realizada a conexão entre estes três arquivos (FILE, ISEA e SIGGRAPH), estaremos criando interconexões recíprocas entre os repositórios, com conteúdos que se reforçam entre si e com o propósito de se ampliar posteriormente esta interconexão com outros arquivos de arte e tecnologia presentes no Brasil e em outros lugares do mundo.

Uma rede mundial de arquivos conectados, disponível de forma on-line e gratuita, e tendo o FILE Festival como um de seus núcleos, permitirá o posicionamento em destaque de uma instituição brasileira, e, logo, do Brasil, dentro do contexto internacional não apenas na continuidade da discussão de estratégias de apoio ao patrimônio cultural digital, mas também na interconexão com as principais instituições culturais da área mundialmente. Conectar o arquivo do FILE numa rede internacional de arquivos que concentram a história da arte e da tecnologia proporciona acesso aos pesquisadores e estudantes brasileiros para que encontrem informações sobre a arte produzida a partir das ferramentas digitais do início do século XXI, em âmbito global, juntamente com informações históricas relacionadas à sua evolução ao longo dos anos. Será possível encontrar trabalhos acadêmicos, resumos, informações sobre cursos e workshops e outros materiais relacionados que, por sua vez, devem inspirar novas pesquisas e desenvolvimento inovador de tecnologias e obras de arte. Além disso, o processo operacional deste projeto tem colocado a equipe de profissionais brasileiros em contato com profissionais internacionais, assim como introduzido internacionalmente produtos e soluções brasileiras na área de software e soluções arquivistas construídas a partir da realidade do país, gerando uma troca de conhecimento efetiva entre pesquisadores durante a sua execução. Frequentemente, essas informações não estão disponíveis em nenhuma biblioteca ou on-line, a não ser nos arquivos 
ISEA, ACM SIGGRAPH e FILE. O conteúdo desses arquivos vai muito além das artes e mostra as contribuições de especialistas do mundo da arte, tecnologia, ciência e humanidades.

Acreditamos que tanto a disponibilização da continuidade de discussões em um ambiente cultural on-line, como a disponibilização de arquivos internacionais e interconectados nesta área, pode democratizar o acesso às informações e ensino, com o intuito de formar um polo para distribuição de informação na área de artes e mídias digitais.

Uma vez que for realizada esta experiência empírica e prova de conceito com a conexão entre estes três arquivos (FILE, ISEA e SIGGRAPH), estaremos criando interconexões recíprocas entre eles, com conteúdos que se reforçam entre si. Dessa forma, poderemos avançar com o propósito de se ampliar posteriormente essa interconexão com outros arquivos de arte e tecnologia presentes em outros lugares do mundo.

\section{Referências bibliográficas}

Bevilaceua, G. M. F. Bancos de Dados e Informatização ed Arquivos: pressupostos teóricos e aplicações técnicas. Monografia (Mestrado em História social) - Faculdade de Filosofia, Letras e Ciências Humanas, Universidade de São Paulo. São Paulo, p. 156, 2010.

DEPOCAS, A.; IPPOLITO, J.; JONES, C. (ed.). L'approche des médias variables: la permanence par le changement, Nova York, Montreal, 2003. Disponível em: <https://www.variablemedia.net/f/preserving/html/var_pub_index.html $\geq$. Acesso em: 25, jul. 2021.

DIAS, C. V. S. M.; MARTINS, D. L. "Iniciativas brasileiras em torno da construção de uma política nacional para acervos digitais de instituições de memória:o desafio da memória em tempos de cultura digital”. Políticas Culturais em Revista. Salvador, v. 13, n. 1, p. 16-46, jan.-jun. 2020. Disponível em: <https://periodicos.ufba.br/index.php/pculturais/article/view/35616>. Acesso em: 18, ago. 2021.

FARIAS, D. S. Mediação da Informação através de plataformas digitais: práticas nas bibliotecas, arquivos e museus da área metropolitana do Porto. Monografia (mestrado em Ciências da Informação) - Faculdade de Engenharia, Universidade do Porto. Porto, p. 78, 2018.

Fino-Radin, B. Digital Preservation Practices and the Rhizome Artbase. 2011. Disponível em: <http://media.rhizome.org/artbase/documents/Digital-Preservation-Practices-and-the-Rhizome-ArtBase.pdf>. Acesso em: 14, set. 2021.

Gannis, C.; Hazrati, M; Meerdo, C. “Recollecting Futures”. Art Reveal, 27 de março de 2021. Disponível em: <https://www.artrevealmagazine.com/recollecting-futures/>. Acesso em: 24, ago. 2021.

GASPARETTO, D. A. Arte digital no Brasil e as (re)configurações no sistema da arte. Tese (Doutorado em Artes Visuais) - Instituto de Artes, Universidade Federal do Rio Grande do Sul, Porto Alegre, p. 289, 2016.

GOBIRA, P. (org.). A memória do digital e outras questões das artes e museologia. Belo Horizonte: EdUEMG, p. $255,2019$.

GOBIRA, P.;MUCELLI, T. (org.). Configurações do pós-digital: Arte e cultura tecnológicas. Belo Horizonte: EdUEMG, p. $313,2017$.

GraU, O. Digital Art through the Looking Glass: New strategies for archiving, collecting andpreserving in Digital Humanities. $1^{\text {a }}$ ed. Donau: Universitat Krem, 2018.

Hofman, V.; Alsina, P. “Art and speculative futures 'What would happen if...?”. Artnodes, n. 19, 2017. Disponível em: <https://raco.cat/index.php/Artnodes/article/view/n19-hofman-alsina >. Acesso em: 21, set. 2021. 
MunSter, A. Materializing new media: embodiment in information aesthetics. Hanover: Dartmouth, 2006,256 p.

ORTH, G. P. "Entre a contingência e a permanência: arquivos nas linguagens eletrônicas”. In: BEIGUELMAN, G.; MAGALHÃES, A. G. (Orgs.). Futuros Possíveis: arte, museuse arquivos digitais. São Paulo: Editora Peirópolis Ltda., 2014.

PERIssinoto, P. (org.). FILEALIVE / ARQUIVOVIVO: Online meetings. White Paper. São Paulo: FILE - International Electronic Language Festival, 2021. Disponível em: <https://drive.google.com/file/d/1Lm_Yl_sV-BkgfHej4J4YNvBdRZGZYIu/view>. Acesso em 23, jul. 2021.

PHILliPs, J. What Is “Time-Based Media”?: A Q\&A with Guggenheim Conservator Joanna Phillips O que é "mídia baseada no tempo"? [Entrevista concedida a] Caitlin Dover. Guggenheim, Nova York, 4 mar. 2014. Disponível em: <https://www.guggenheim.org/blogs/checklist/what-is-time-based-media-a-q-and-a-with-guggenheim-conservatorjoanna-phillips >. Acesso em: 5, jul. 2021.

RINEHART, R.; IPPOLITO, J. Re-collection:Art, New Media, and Social Memory. Massachusetts: MIT Press, p. 297 , jun. de 2014.

VERAS, L. (org.). Abre-te Código - Transformação Digital e Patrimônio Cultural. São Paulo: Goethe Institut, 2020. Disponível em: <https://issuu.com/bdlf/docs/upload_this_e-book_abre-te>. Acesso em: 14, maio, 2021.

\section{Palestras e Webinars}

Conservation Center - NYU Institute Of Fine Arts. Experiential Conservation: The Media Preservation Initiative Model at the Whitney with Carol Mancusi-Ungaro, Dr. Chrissie Iles, Farris Wahbeh, David Neary, Savannah Campbell, Brian Block, Christopher Bernu, and Nicholas Carbone. Disponível em: <https://www.ifa.nyu.edu/conservation/timebased-media.htm >. Acesso em: 16, jun. 2021.

FILEALIVE/ARQUIVOVIVO. Arquivo como Instituição. Disponível <https://www.youtube.com/watch?v=VE63Yoa118M>. Acesso em: 23, jul. 2021. 2021a.

O que arquivar? Demandas e princípios da memória digital. Disponível em: <https://www.youtube.com/watch?v=4LCQXsssdqI>. Acesso em: 23, jul. 2021. 2021b.

New York University - Abu Dhabi ART Gallery. An Oral History of the Early Internet: After 40 years of cyberspace, what is left of the utopian dream? Disponível em: <https://www.nyuad-artgallery.org/en_US/resources/watch/an-oralhistory-of-the-internet/>. Acesso em: 25, ago. 2021.

University Of Maine. Digital Curation Teleconference with Anna Perricci. Disponível em: $<$ https://digitalcuration.umaine.edu/teleconferences/dig_perricci_teleconf_2020.html>. Acesso em: 8, ago. 2021.

\section{Sites citados}

Ars Electronica - <https://ars.electronica.art/>

Digital Art Archive - <https://www.digitalartarchive.at/>

Digital Art Archive - SIGGRAPH - <https://digitalartarchive.siggraph.org/>

FILE - <https://file.org.br/> 
FILEALIVE / ARQUIVOVIVO - <https://alive.file.org.br/>

ISEA Archives - <http://www.isea-archives.org/>

ISEA Archives -SIGGRAPH - <https://isea-archives.siggraph.org/>

Media Art Research - <http://mediaartresearch.org/search/thesaurus-hie- rarchical.html>

Strapi - <https://strapi.io/>

Tainacan - <https://tainacan.org/>

Tainacan, Artigos - <https://pesquisa.tainacan.org/tipo-pos-graduacao/artigos/>

W3Techs $-<$ https://w3techs.com/>

WikiData - <https://www.wikidata.org/wiki/>

ZKN - <https://zkm.de/> 